\title{
Pupillary changes in two memory tasks'
}

Five $S$ s recalled telephone numbers from long-term and short-term memory while pupil diameter was measured. Major pupillary changes were found in both tasks. Pupil diameter seems to vary with momentary load on $S$.

Hess \& Polt (1964; Hess, 1965) have reported that the pupil of the eye dilates during the solution of arithmetic problems, and that the extent of dilation is related to the difficulty of the problem. More recently, Kahneman \& Beatty (submitted) showed that pupil diameter is linearly related to the amount of materlal stored for immediate recall in a paced digit-span task. The pupil dilates as $S$ listens to a string of digits or words (the loading function) and constricts as he reports these items from short-term memory (the unloading function). The amount of dilation was related to both the length of the string and the difficulty of the task. These results suggested that pupil diameter may have construct validity as a measure of the momentary "load" on $S$ as he performs a mental task. The present study was designed to compare the loading and unloading functions in a long-term memory task with those observed in short-term tasks.

Method

Five undergraduates (two males) served as Ss. Before the experimental session, each $\mathrm{S}$ provided a list of five or six telephone numbers with which he was thoroughly familiar, together with a brief name for each number. To test recall from long-term memory, E said one of these names, and $S$ after a brief pause produced the number associated with it. To test short-term memory, E said a number (taken from the list supplied by another $S)$ and $S$ repeated that number. Pictures of the pupil were obtained at $1 / \mathrm{sec}$, during each trial.

$S$ was positioned in a chin/forehead rest, looking at a fixation surface $10 \mathrm{in.}$ away. A television camera, with a $100 \mathrm{~mm}$ telephoto lens and extension tubes, was focused on S's right eye. A half-silvered mirror was placed before $S$, so that the television camera was not in his field of view. The image of S's eye was displayed on a monitor, adjusted to provide maximum contrast between iris and pupil. Photographs of this image were made with a Grass C4D Kymograph camera on $35 \mathrm{~mm}$ Tri-X film. Pictures were taken at the rate of $1 / \mathrm{sec}$. throughout each trial. The click of the Grass camera was audible enough to serve as a pacing device for both $\mathrm{E}$ and $\mathrm{S}$. Actual measurements of pupil diameter were later made from projections of this film. In a test of reliability among readers, the standard error of measurement was found to be $0.042 \mathrm{~mm}$.

On long-term memory trials, the $S$ was given a ready signal, and the camera began taking pictures at the rate of $1 / \mathrm{sec}$. On the fifth picture, $\mathrm{S}$ was given the name which he had supplied for one of the telephone numbers. After a pause of $2 \mathrm{sec}$, he began repeating the telephone number in time with the camera, one digit on each click. Three additional pictures were taken after $S$ had finished speaking. On short-term memory trials, an unfamiliar telephone number was presented to $S$ during a $3 \mathrm{sec}$. period, beginning on the fifth picture and concluding by the seventh. $S$ then waited 2 more sec. before responding as before. The only difference in timing between the types of trials is that stimulus presentation in the short-term memory task took $2 \mathrm{sec}$. longer. In all other respects the trials were identical. As a check on the synchrony of E's presentation and S's responses with the timing of the pictures, all three events were recorded on a Bausch and Lomb VOM-5 strip chart recorder. No errors of timing were found. Four trials of each type were given to $S$ in random order. Any trial in which an error occurred was later replaced. Resulis

The results of the experimentare presented in Fig. 1. Essentially similar results were obtained for all Ss. In the short-term condition, the pupil dilates during intake of information, dilates slightly more during the pause, and constricts during the report, reaching baseline level on the last digit spoken. These results are essentially identical to the findings in a standard test of immediate retention of digits (Kahneman \& Beatty, submitted). Again, both the dilation and the constriction follow essentially linear trends in time. In the long-term condition, the presentation of the verbal cue to which $\mathbf{S}$ responds with a number occasions an extremely rapid dilation (approximately $8 \%$ of baseline diameter c'uring

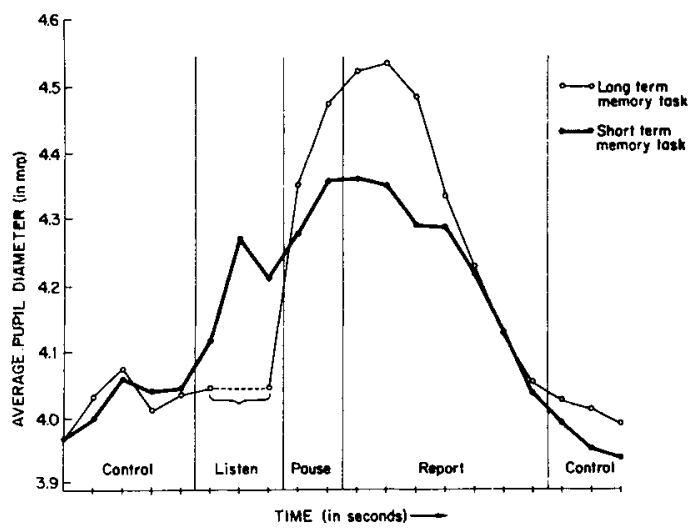

Fig. 1. Average pupil diameter for five Ss during presentation and report of seven-digit telephone numbers from short-term and long-term memory. The long-term memory function is broken above the brace, with both points representing the same pupillary measurements. 
the first sec.). The peak pupillary diameter attained is consistently higher in the long-term than in the short-term condition $(t=6.77, \mathrm{df}=4, \mathrm{p}<.01)$, but the constriction during the report is very similar under the two conditions.

\section{Discussion}

The suggestion has been advanced by Hess \& Polt (1964) that pupil diameter may serve as an index of mental activity. Kahneman \& Beatty (submitted) presented additional evidence that the pupil is surprisingly sensitive to the momentary load on S: the pupil appeared to follow, second by second, the amount of effort required by the short-term memory tasks which were investigated. The present study attempted to extend this conclusion to a task involving retrieval from more permanent memory; obviously, an effort is exerted by the individual as he attempts to retrieve the telephone number associated with the cue which he has heard. Presumably, this effort is maximal very shortly after the presentation of the cue, as the number is recalled and the verbal response is organized. The rapid dilation of the pupil under these conditions is therefore important evidence for the construct validity of changes of pupil diameter as a measure of the momentary state of mental effort.

Hess \& Polt (1964) had shown that the peak pupillary dilation obtained during the solution of an arithmetic problem is related to the difficulty of the problem. Kahneman \& Beatty confirmed and extended this finding, showing that peak pupillary diameter is directly related to the length of a string of digits which $\mathrm{S}$ is to report, that words (which are more difficult to recall) occasion larger dilations than the same number of digits, and that very large dilations a re obtained when $S$ is to trans- form a string of digits prior to report. By the characteristically circular logic of construct validation (Cronbach \& Meehl, 1955), it now appears permissible to infer that the effort required in retrieving even a thoroughly familiar telephone number on cue is quite substantial. Our data suggest that this effort is considerably greater than the effort required to repeat a series of seven digits after a 2-sec. pause. However, it should not be concluded from these results that retrieval of information from long-term memory will invariably elicit a greater pupillary response than short-term storage and recall of an equivalent message. For example, a study of pupillary changes during a verbal learning task (Kahneman \& Beatty, in preparation) showed that the pupillary responses associated with recall of a response diminish consistently as a simple association is overlearned. Presumably, requesting the same number twice in rapid succession would have occasioned smaller pupillary responses in the present experiment as well.

\section{References}

Cronbach, L. J., \& Meehl, P. E. Construct validity in psychological tests. Psychol. Bull., 1955, 52, 281-300.

Hess, E., \& Polt, J. Pupil size in relation to mental activity during simple problem-solving. Science, 1964, 140, 1190-1192.

Hess, E. Attitude and pupil size. Scient. American, 1965, 212. 46-54.

Kahneman, D., \& Beatty, J. Pupil diameter: A linear relationship with memory load. (Submitted).

\section{Note}

1. This research was supported in part by Grant NIMH No. 08847-02 to G. S. Blum. We thank Dr. I. Pollack of the Mental Health Research Institute of the University of Michigan, who provided the facilities. 